БЫСТРЫЕ ЗВЕЗДНЫЕ ПУЛЬСАЦИИ

И ЛОКАЛЬНЫЕ МАГНИТНЫЕ ПОЛЯ

\author{
Холтыгин А.Ф. ${ }^{1}$, Моисеева А.В. ${ }^{2}$, Якунин И.А. ${ }^{2}$, \\ Циопа О.А. ${ }^{3}$, Валеев А.Ф. ${ }^{2}$ \\ ${ }^{1}$ Санкт-Петербургский государственный университет, Санкт-Петербург, Россия \\ ${ }^{2}$ Специильная астрофизическая обсерватория РАН, Нижний Архыз, Россия \\ ${ }^{3}$ Главная (Пулковская) астрономическая обсерватория РАН, Санкт-Петербург, Россия
}

\title{
FAST STELLAR PULSATIONS AND LOCAL MAGNETIC FIELDS
}

Kholtygin A.F. ${ }^{1}$, Moiseeva A.V. ${ }^{2}$, Yakunin I.A. ${ }^{2}$, Tsiopa O.A. ${ }^{3}$, Valeev A.F. ${ }^{2}$

${ }^{1}$ St. Petersburg State University, St. Petersburg, Russia

${ }^{2}$ Special Astrophysical Observatory of the RAS, Nizhny Arkhyz, Russia

${ }^{3}$ Main (Pulkovo) Astronomical Observatory RAS, St. Petersburg, Russia

The results of studies by Kholtygin et al. [1-3] of superfast (on minute and second scales) line profile variability in spectra of early-type star are reviewed. The spectra of program OBA stars were obtained with the 6-meter BTA telescope using a multi-mode SCORPIO reducer and using the MSS spectrograph with an exposure of 1-150 seconds and a total observation time of 2-3 hours. The variability of the line profiles is analyzed. Regular variations of the H, HeI, FeII, NII, OII, SiII line profiles were detected in spectra of program stars with periods from 2 to 120 minutes and amplitudes of 1-2\% of the continuum level. The frequencies of the line profile variations in the spectra of some stars with periods less than 10 minutes appeared to be variable. The frequencies of the short-period components can change by $20 \%$ during the observation period possibly and have a nature close to the nature of five-minute solar oscillations. We proposed a connection between irregular line profile variations with local magnetic fields and generated by them stellar micro-flares.

\section{DOI: 10.31725/0552-5829-2020-317-320}

\section{1. Наблюдения и обработка}

Настоящая статья посвящена исследованию сверхбыстрой переменности профилей линий в спектрах ярких ОВА звезд. Наблюдения были выполнены в 2015-2017 гг. на 6-м телескопе БТА со спектрографом низкого разрешения SCORPIO, в 2019-2020 гг. со спектрографом ОЗСП и на 1.25 м телескопе Крымской станции ГАИШ МГУ. Первичная редукция ПЗС-изображений спектров выполнена в средах Midas и IRAF. Было получено почти 8000 спектров 14 ОВА звезд (см. список звезд в статье [4]). Все полученные спектры были нормированы на уровень континуума. Для иллюстрации на рис. 1 представлен средний спектр химически пекулярной звезды А0Vp звезды $\alpha^{2} \mathrm{CVn}$. 


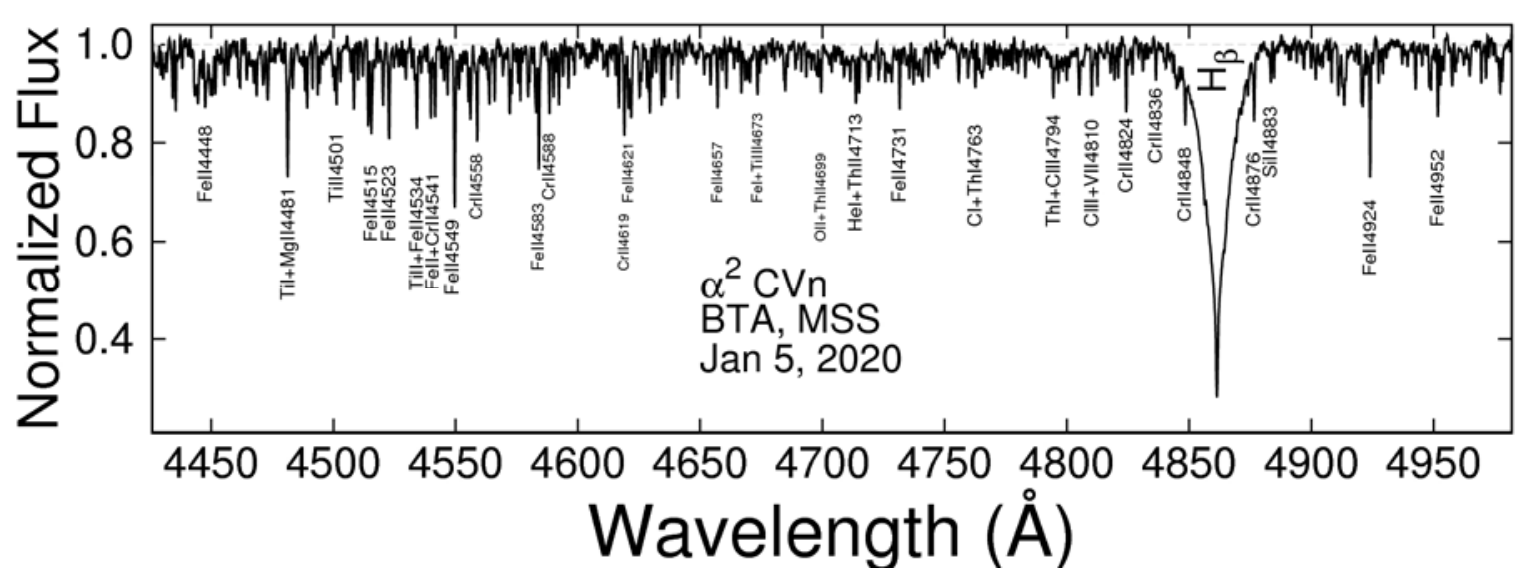

Рис. 1. Средний спектр звезды $\alpha^{2} \mathrm{CVn}$

\section{2. Регулярная переменность профилей}

Для исследования переменности профилей были проанализированы разностные профили

$$
d\left(V, t_{i}\right)=F_{i}(V)-\bar{F}_{i}(V),
$$

где $F_{i}(V)$ - нормированный на континуум поток в линии в спектре с но-

мером $i$, соответствующий доплеровскому смещению $V$, а $\bar{F}_{i}(V)-$ средний по всем наблюдениям поток бальмеровских линий водорода, линий $\mathrm{HeI}$ и других ионов в спектрах всех программных звезд (см. рис. 1). Фурье-анализ временных рядов разностных профилей линий, соответствующих всем моментам наблюдений $t_{\mathrm{i}}$, показал присутствие регулярных компонентов вариаций профилей линий в спектрах всех исследуемых звезд с периодами 10-120 минут.

\section{3. Оконное преобразование Фурье и сверхбыстрые вариации профилей}

Для поиска компонентов вариаций профилей с периодами меньше 10 минут было использовано оконное Фурье-преобразование:

$$
F\left(t_{0}, \Delta t, v, V_{k}\right)=\int_{-\infty}^{+\infty} d(t) W\left(\tau-t_{0}, \Delta t\right) e^{-i 2 \pi v \tau} d \tau
$$

где $t_{0}$ - начало окна, а $\Delta t$ - его ширина. В качестве оконной функции $W(t, \Delta t)$ используется функция Хемминга [5]. Для полного профиля линии оконное преобразование является функцией 4-х переменных, поэтому мы просуммировали значения указанной функции для всех доплеровских смещений $V_{k}$ в пределах профиля линии и зафиксировали ширину окна $\Delta t$.

Результаты применения оконного Фурье-преобразования к вариациям профиля линии $\mathrm{H}_{\delta}$ в спектре звезд $\alpha^{2} \mathrm{CVn}(\mathrm{AOVp})$ и $\gamma \mathrm{UMi}(\mathrm{A} 2 \mathrm{III})$ представлены на рис. 2. В области частот $v>0.1 \mathrm{~min}^{-1}$ видны транзиентные 
компоненты вариаций профилей, соответствующие периодам. Компонент (1) в оконном Фурье-спектре $\alpha^{2} \mathrm{CVn}$ (рис. 2, справа) имеет длительность $\sim 40$ минут, период этого компонента меняется от 5.5 до 4.8 минут. Компонент (2) в оконном Фурье-спектре $\gamma$ UMi длительностью $~ 30$ минут характеризуется постоянным периодом 9.6 минут в согласии с отмеченным в статье [3] увлечением периода быстрых вариаций профилей линий при переходе к звездам более поздних классов.
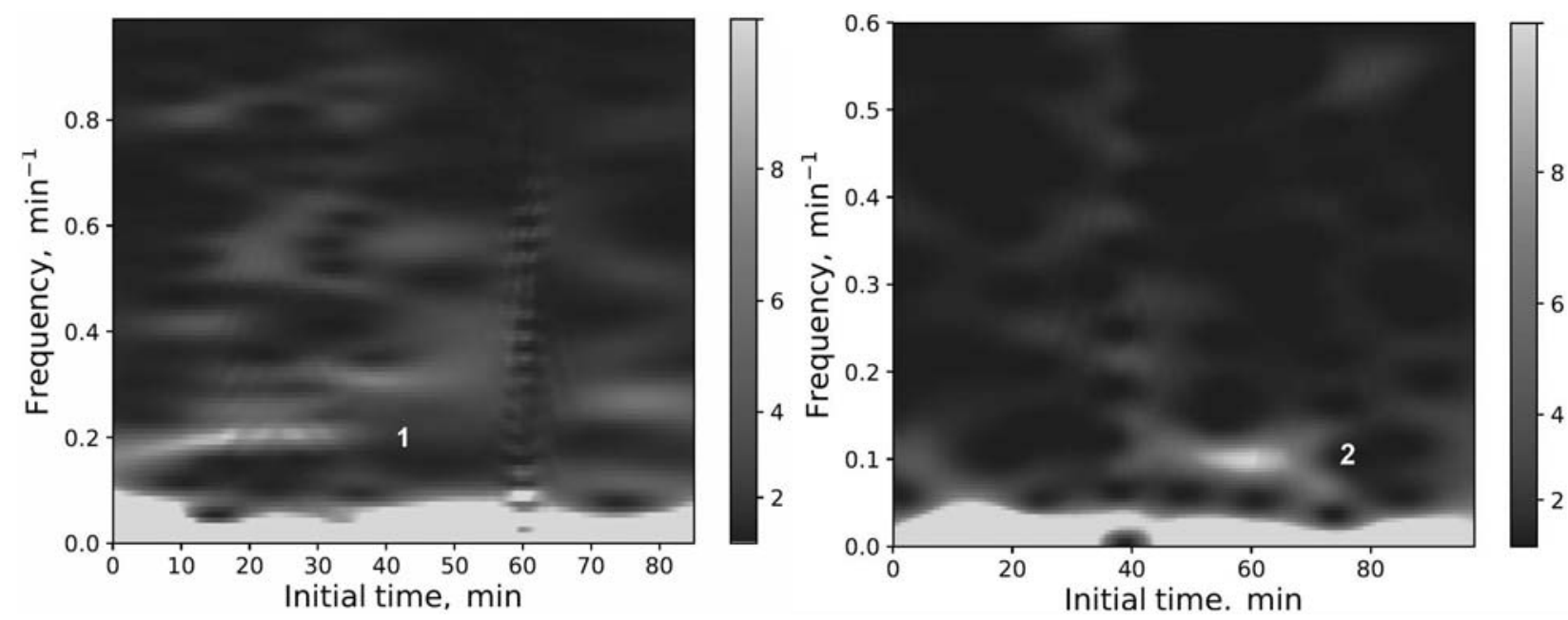

Рис. 2. Оконное преобразование вариаций профиля линии $\mathrm{H}_{\delta}$ в спектре звезды $\alpha^{2} \mathrm{CVn}$ (слева) и $\gamma \mathrm{UMi}($ справа).

Кроме уже отмеченных, на рис. 2 видно много слабых транзиентных компонентов, соответствующих периодом 1-5 минут. Быстрые вариации профилей с периодами меньше 10 минут ранее не были известны и, вероятнее всего, связаны с высоким модами нерадиальных пульсаций (НРП, см., например [1]). Быстрые пульсации с периодами 1-10 минут характерны для OB субкарликов, roАp звезд и звезд солнечного типа [7].

Частоты обнаруженных нами компонентов вариаций профилей соответствуют частотам высоких $(l=200-1000)$ мод нерадиальных пульсаций (НРП) на Солнце (например, [6]). Применимость соотношений между частотами и модами НРП, представленных в работе [6] для ОВА звезд главной последовательности требует дополнительного анализа.

Исходя из стандартных масштабных соотношений между частотами НРП и значениями $l$ (например, [7]) можно сделать вывод, что для звезд ранних спектральных классов пульсации с периодами меньше 10 минут соответствуют значениям $l>1000$. Столь высокие моды НРП могут быть нестабильными и короткоживущими, что может объяснить транзиентный характер обнаруженных регулярных вариаций профилей линий в спектрах ОВА звезд и дрейф их частот (периодов).

Кроме регулярных компонентов вариаций профилей в спектрах звезд HD 93521 (O9.5III) и $\rho$ Leo (B1Iab) зарегистрированы нерегулярные вари- 
ации на минутных шкалах времени [1-2], подобные обнаруженным в профиле линии НеI 6678 в спектре Ве звезды $\lambda$ Eri [8]. Такие стохастические вариации могут быть результатом оптического послесвечения микровспышек, связанных с локальными магнитными полями на этих звездах. Методика [9] детектирования подобных полей у звезд спектральных классов K и $\mathrm{G}$ может быть использована для поиска таких полей у ОВА звезд.

\section{4. Заключение и выводы}

В результате выполнения нашей программы поиска сверхбыстрой переменности профилей было получено почти 8000 индивидуальных спектров программных звезд. На основании анализа выполненных наблюдений можно сделать следующие выводы:

Обнаруженные вариации профилей в основном имеют регулярный характер с периодами от 1 до $\sim 300$ минут и могут быть связаны с высокими модами нерадиальных пульсаций (НРП).

Вариации профилей в интервале частот $v<0.1$ мин $^{-1}$ имеют транзиентный характер со временем жизни от 30 до 120 минут. Периоды регулярных компонентов таких вариаций могут меняться на 10-20\% за время жизни. Такое поведение можно объяснить нестабильностью мод НРП с $l>1000$.

\section{Благодарности}

Настоящее исследование поддержано грантом РФФИ 19-02-00311 А.

\section{Литература}

1. Kholtygin A.F., S. Hubrig, V.V. Dushin, et al. // ASP Conf. Ser., 2017, 510, 299.

2. Kholtygin A.F., A.A. Batrakov, S.N. Fabrika, et. al. // Astroph. Bull., 2018, 73, 471.

3. Kholtygin A.F., N.P. Ikonnikova, A.V. Dodin, O.A. Tsiopa // Astron. Lett., 2020, 46, 168.

4. Kholtygin A.F., et al. // ASP Conf. Ser., in press (2020).

5. The Handbook of Formulas and Tables for Signal Processing, Ed. A.D. Poularikas, Boca Raton: CRC Press LLC, 1999.

6. Korzennik S.G., M.C. Rabello-Soares, J. Schou, T.P. Larson // ApJ, 2013, 772, 87.

7. Di Mauro M.P. Frontier Research in Astrophysics II, 2016.

8. Smith M.A., R.S. Polidan // ApJ, 1993, 408, 323.

9. Плачинда С.И., В.В. Бутковская // Изв.Крымск.Астрофиз.Обсерв., 2020, 116, 45. 